\title{
Traditional Chinese medicine baoxin decoction improves cardiac fibrosis of rats with dilated cardiomyopathy
}

\author{
RONGRONG SUN ${ }^{1 *}$, JIANGBO WANG $^{2 *}$, YI ZHENG $^{3}$, XIANCHI $^{3}{ }^{3}$, TIANTIAN XIE $^{3}$, RUI LI $^{3}$, \\ MIN LIU ${ }^{4}$, YONG CAO ${ }^{4}$, LEI LU ${ }^{4}$, QING ZHANG ${ }^{4}$ and PEIYING ZHANG 5 \\ ${ }^{1}$ Clinical Medicine, Nanjing University of Traditional Chinese Medicine, The Affiliated Xuzhou Central Hospital \\ of Nanjing University of Traditional Chinese Medicine, Nanjing, Jiangsu 210023; Departments of ${ }^{2}$ Neurology \\ and ${ }^{3}$ Cardiology, Xuzhou Central Hospital; ${ }^{4}$ Department of Cardiology, Xuzhou Hospital of Traditional Medicine, \\ ${ }^{5}$ Department of Cardiology, The Affiliated Xuzhou Central Hospital of Nanjing University of Traditional Chinese Medicine, \\ The Affiliated Xuzhou Hospital of Medical School of Southeast University, Xuzhou, Jiangsu 221009, P.R. China
}

Received December 5, 2016; Accepted January 27, 2017

DOI: $10.3892 /$ etm.2017.4223

\begin{abstract}
We investigated the effect of baoxin decoction (BXD) on myocardial fibrosis and clarified the possible mechanism of action. Dilated myocardiopathy was induced by doxorubicin injected intraperitoneally for 6 weeks. Rats that demonstrated dilated myocardiopathy were randomly divided into five groups plus a control group. Three groups were treated with BXD $(7.5 / \mathrm{kg}, 15 \mathrm{~g} / \mathrm{kg}$ and $30 \mathrm{~g} / \mathrm{kg})$ daily for 4 weeks. One group was treated with $8.75 \mathrm{~g} / \mathrm{kg}$ of captopril (positive control), and with physiologic saline (negative control). Cardiac function was evaluated using echocardiography. Hematoxylin and eosin, and Masson's trichrome staining were performed, PICP and PIIINP were assessed by ELISA, the expression of galectin-3 and collagen types I and III was evaluated with reverse transcription-quantitative PCR, and interrelated proteins were detected by western blot analysis. BXD downregulated galectin-3, collagen I and III and was correlated with a high expression of fibrosis markers. It also significantly decreased myocardial collagen volume fraction $(\mathrm{CVF})$, together with markedly preventing the upregulation of collagen I and III. In addition, BXD downregulated the expression of TGF- $\beta 1$ and Smad3 in the myocardial fibrosis rats. Therefore, BXD treatment significantly improved cardiac function and alleviated myocardial fibrosis in a rat model of
\end{abstract}

Correspondence to: Professor Peiying Zhang, Department of Cardiology, The Affiliated Xuzhou Central Hospital of Nanjing University of Traditional Chinese Medicine, The Affiliated Xuzhou Hospital of Medical School of Southeast University, 199 South Jiefang Road, Xuzhou, Jiangsu 221009, P.R. China

E-mail: zpying58@126.com

*Contributed equally

Key words: traditional Chinese medicine, baoxin decoction, cardiac fibrosis, dilated cardiomyopathy doxorubicin-induced dilated cardiomyopathy (DCM), which is the mechanism that may be associated with inhibiting the TGF- $\beta 1$ signaling pathway.

\section{Introduction}

Dilated cardiomyopathy (DCM) is a common cardiomyopathy leading to heart failure (HF), and is characterized by the enlargement of one or both of the ventricles with associated systolic dysfunction (1). In adults, the prevalence is $\sim 1$ in 2,500 individuals, with an incidence of 7/100,000 per year; however, it maybe underdiagnosed. The prevalence of DCM in the United States (adjusted for age) is 36/100,000 of the population (2). DCM is an important cause of sudden cardiac death (SCD) and HF and is the leading indication for cardiac transplantation in children and adults worldwide (3).

Ventricular remodeling is the key process of DCM and HF pathogenesis, and was originally referred to an alteration in the ventricular architecture (4). It is characterized by a structural rearrangement of the cardiac chamber wall that involves not only cardiomyocyte hypertrophy, but also the fibroblast proliferation, and an increased deposition of extracellular matrix (ECM) proteins (5). It refers to the aberrant deposition of ECM in the heart, and is characterized by an increase of collagen in the interstitium. As shown experimentally, an increase in collagen content increases myocardial stiffness and promotes abnormalities of cardiac function, whereas its regression normalizes stiffness and function (6). Myocardial fibrosis is an important pathophysiological process in which the accumulation of collagen contributes to DCM and HF (7). To understand the components of myocardial fibrosis and remodeling, current medication has been used to counteract the compensatory mechanism of ventricular remodeling and, consequently, to reduce morbidity and mortality (8).

Traditional Chinese medicine (TCM) has been applied to treat HF for thousands of years, and some herbal formulas have been proven to be effective (9). Baoxin decoction (BXD), is a compound that has been prepared and formulated on the basic theory of TCM, and consists of a complex combination of natural herbs where every herb contains various chemical 
compounds (10). These compounds can work together in mutual coordination and auxiliary, which has been used to treat DCM and leads to HF. Clinical studies have demonstrated that BXD can improve myocardial dysfunction in DCM patients. In the present study, we aimed to examine the hypothesis that BXD exerts its cardioprotective effect by preventing myocardial fibrosis in a rat model of doxorubicin-induced DCM.

\section{Materials and methods}

Drug preparation. Traditional Chinese herb granules manufactured by Guangdong Yifang Pharmaceutical Co., Ltd. (Guangdong, Guangzhou, China) were purchased from the pharmacy of Traditional Chinese Medicine Hospital. The granules were prepared from Astragalus $(30 \mathrm{~g})$, Salvia miltiorrhiza (10 g), Cassia twig (6 g), Wolfiporia extensa (10 g), Forsythia suspense (10 g), Turtle shell (10 g), Angelica sinensis (10 g), Codonopsis pilosula (10 g), Ophiopogon japonicus (10 g) and Schisandra chinensis (6 g). After preparation, the herbal mixtures were freeze-dried. Prior to use, each $2 \mathrm{~g}$ of dry herbal mixture was resuspended in $1 \mathrm{ml}$ of distilled water.

Experimental animals. Male Sprague-Dawley rats $(\mathrm{n}=70)$ weighing 220 $\pm 10 \mathrm{~g}$, were purchased from the Laboratory Animal Center of Xuzhou Medical College [Xuzhou, Jiangsu, China, license no. SCXK (Su) 2010-0003]. The rats were housed in a normal 12-h light/dark rhythm under standard conditions of temperature $\left(21 \pm 2^{\circ} \mathrm{C}\right)$ and humidity $(55 \pm 5 \%)$ and fed with a standard diet and tap water. Animals were allowed a 1-week acclimatization period prior to the experimental protocol. The animal care, use and experimental protocols were approved by the Animal Experimental Ethics Committee of Xuzhou Medical University.

Animal models. A total of 62 animals were subjected to intraperitoneal injections of doxorubicin hydrochloride (Zhejiang Haizheng Pharmaceutical Industry, Zhejiang, China) $2.5 \mathrm{mg} / \mathrm{kg}$ weekly for 6 consecutive weeks (11). Eight rats received the same volume of physiological saline as control. Two weeks after cessation of the doxorubicin injection, the two groups of rats that survived were examined by transthoracic echocardiography to determine whether the model was successful.

Grouping and treatment. With the exception of the control animals, the surviving animals developed ventricular dysfunction with a left ventricular ejection fraction (LVEF) of $<60 \%$ $(n=42)$. The rats were randomly divided into five groups: the DCM group (DCM, n=9, saline), the low-dose BXD group (BXD-L, n=9, 7.5/kg), the middle-dose BXD group (BXD-M, $\mathrm{n}=8,15 \mathrm{~g} / \mathrm{kg}$ ), the high-dose BXD group (TXL-H, $\mathrm{n}=8$, $30 \mathrm{~g} / \mathrm{kg}$ ) and the positive group ( $\mathrm{n}=8$, captopril, $8.75 \mathrm{~g} / \mathrm{kg}$ ). Doses were selected on the basis of human clinical dosage and the control group and DCM group rats were administered saline.

Echocardiography measurements. Before and after treatment, transthoracic echocardiography was performed in all the groups. The rats were anesthetized using chloral hydrate. Images were captured using a $12 \mathrm{MHz}$ linear transducer connected to a Vivid 7 echocardiography machine (GE Healthcare, Aurora, OH, USA). A two-dimensional short-axis view of the left ventricle was obtained at the level of the papillary muscle and two-dimensional targeted M-mode tracings were recorded. The detection index was as follows: left ventricular ejection fraction (LVEF), left ventricular end-diastolic diameter (LVEDD), left ventricular end-systolic diameter (LVESD) and left ventricular fractional shortening (LVFS). All the parameters were measured over three consecutive cardiac cycles.

Measurement of serum indicators by ELISA. Blood samples were collected from the abdominal artery and the serum was separated. Procollagen type I carboxy-terminal peptide (PICP) and procollagen type III aminoterminal peptide (PIIINP) in serum were determined by enzyme-linked immunosorbent assay (ELISA) kits (hCG ELISA kit, Ontario, Canada) following the manufacturer's instructions.

Pathology and histology. Left ventricular tissues of the same region were fixed in $4 \%$ paraformaldehyde. Paraffin-embedded tissues were sectioned into slices $(4 \mu \mathrm{m})$ and stained with hematoxylin and eosin staining (H\&E). Masson's trichrome staining was performed to assess the degree of myocardial fibrosis according to protocol (12). In the Masson's-stained sections, myocardial cells were stained red while the collagen was stained blue. Six randomly selected microscopic fields of each section were analyzed for collagen deposition using Image-Pro Plus 6.0 (Media Cybernetics, Inc., Rockville, MD, USA), which was expressed as collagen volume fraction (CVF), the percentage of the area stained blue for collagen to the total area of each microscopic field. The CVF of each animal represents the mean of 6 randomly selected microscopic fields.

$R N A$ extraction and $R T-q P C R$. Quantitative analysis of the mRNA levels of target genes was performed with RT-qPCR by the relative standard curve method using the SYBR-Green PCR Master mix (Promega, Madison, WI, USA). Total RNA was extracted from frozen ventricular tissue samples using a TRIzol reagent (Takara Bio, Inc., Otsu, Japan). Reverse transcription of RNA was carried out according to the instructions of RT-PCR kit (Promega). The primer sequences used were as follows: galectin-3 (Gal-3) forward, 5'-CCCAACGCAAACA GTATA-3' and reverse, 5'-TGTCTTTCTTCCCTTCCC-3'; collagen I (Col I) forward, 5'-GCCAAGAAGACATCCCT GAA-3' and reverse, 5'-CTTCTGGGCAGAAAGGA CAG-3'; collagen III (Col III) forward, 5'-GGTGGCTTTCAGTTCAG CTATG-3' and reverse, 5'-GTCTTGCTCCATTCACCAG TGT-3'; GAPDH forward, 5'-CTGCACCACCAACTGCT TAG-3' and reverse, 5'-GGATG CAGGGATGATGTTCT-3'.

Western blot analysis. Total protein was extracted from frozen heart tissue by RIPA buffer containing $1 \mathrm{mM}$ PMSF, followed by determination of the protein concentrations with a BCA protein assay kit. The protein was separated using a $12 \%$ SDS polyacrylamide gel in electrophoresis sample buffer and then transferred onto a PVDF membrane. Following washing and blocking, the membrane was incubated overnight at $4^{\circ} \mathrm{C}$ with mouse monoclonal Gal-3 antibody (dilution, 1:500; cat. no. sc-25279), rabbit polyclonal TGF- $\beta 1$ antibody (dilution, 
Table I. LVEDD, LVESD, LVEF and LVFS in the different subgroups of patients.

\begin{tabular}{lllll}
\hline Group & LVEDD & LVESD & LVEF & LVFS \\
\hline CON & $6.14 \pm 0.19^{\mathrm{a}}$ & $3.69 \pm 0.14^{\mathrm{a}}$ & $83.09 \pm 5.33^{\mathrm{a}}$ & $45.86 \pm 3.80^{\mathrm{a}}$ \\
DCM & $7.80 \pm 0.35^{\mathrm{b}}$ & $4.92 \pm 0.23^{\mathrm{b}}$ & $53.42 \pm 3.83^{\mathrm{b}}$ & $26.09 \pm 2.77^{\mathrm{b}}$ \\
BXD-L & $7.43 \pm 0.24^{\mathrm{b}, \mathrm{c}}$ & $4.58 \pm 0.19^{\mathrm{b}, \mathrm{c}}$ & $61.09 \pm 4.80^{\mathrm{a}, \mathrm{b}}$ & $30.18 \pm 1.73^{\mathrm{b}, \mathrm{c}}$ \\
BXD-M & $7.27 \pm 0.24^{\mathrm{a}, \mathrm{b}}$ & $4.52 \pm 0.26^{\mathrm{a}, \mathrm{b}}$ & $64.45 \pm 3.75^{\mathrm{a}, \mathrm{b}}$ & $31.61 \pm 2.09^{\mathrm{a}, \mathrm{b}}$ \\
BXD-H & $7.14 \pm 0.27^{\mathrm{a}, \mathrm{b}}$ & $4.41 \pm 0.22^{\mathrm{a}, \mathrm{b}}$ & $67.07 \pm 3.56^{\mathrm{a}, \mathrm{b}}$ & $34.86 \pm 1.32^{\mathrm{a}, \mathrm{b}}$ \\
CAP & $7.04 \pm 0.32^{\mathrm{a}, \mathrm{b}}$ & $4.36 \pm 0.33^{\mathrm{a}, \mathrm{b}}$ & $67.95 \pm 2.96^{\mathrm{a}, \mathrm{b}}$ & $35.21 \pm 1.49^{\mathrm{a}, \mathrm{b}}$
\end{tabular}

LVEDD, left ventricular end-diastolic diameter; LVESD, left ventricular end-systolic diameter; LVEF, left ventricular ejection fraction; LVFS, left ventricular fractional shortening. ${ }^{\mathrm{a}} \mathrm{P}<0.01$ vs. the $\mathrm{DCM}$ group; ${ }^{\mathrm{b}} \mathrm{P}<0.01 \mathrm{vs}$. the $\mathrm{CON}$ group; ${ }^{\mathrm{c}} \mathrm{P}<0.05$ vs. the $\mathrm{DCM}$ group.
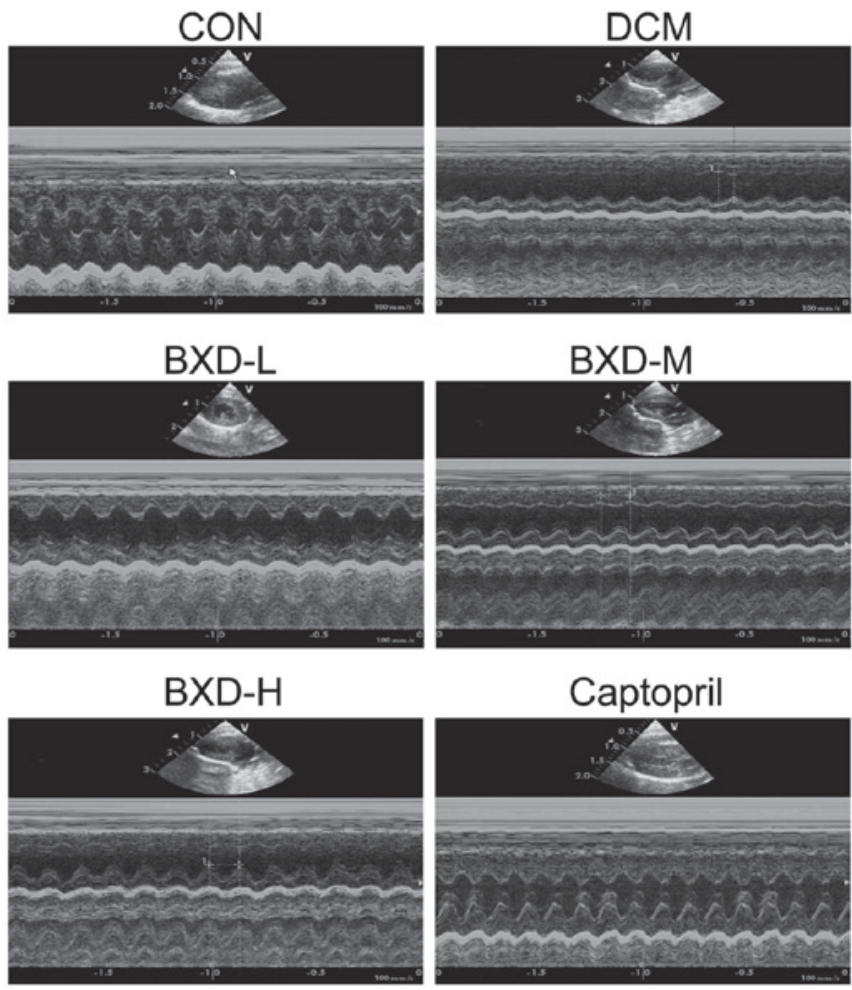

Figure 1. Representative echocardiographic M-mode images. The effect of BXD on the quantitative data of echocardiography images. DCM, dilated cardiomyopathy; BXD, baoxin decoction.

1:500; cat. no. sc-7892), mouse monoclonal Smad3 antibody (dilution, 1:500; cat. no. sc-101154) (all from Santa Cruz Biotechnology, Inc., Santa Cruz, CA, USA). After washing, the membrane was incubated with bovine anti-mouse secondary antibody (dilution, 1:2,000; cat. no. sc-2371; Santa Cruz Biotechnology, Inc.) at $37^{\circ} \mathrm{C}$ for $1 \mathrm{~h}$. After washing, the membrane was incubated with a secondary antibody at $37^{\circ} \mathrm{C}$ for $1 \mathrm{~h}$. A chemiluminescence detection system was used to detect the western blot analysis. Target proteins levels were normalized by GAPDH.

Statistical analysis. Data are presented as mean \pm SD. Statistical analysis was performed using a one-way analysis of variance (ANOVA). $\mathrm{P}<0.05$ was considered to be statistically significant.
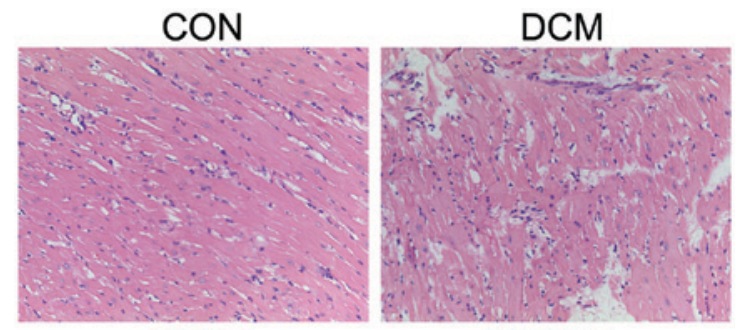

BXD-L

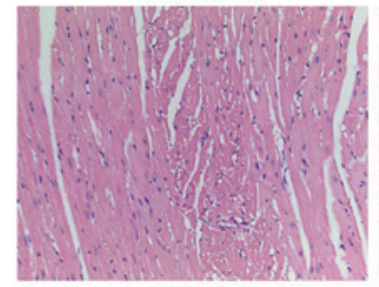

BXD-H
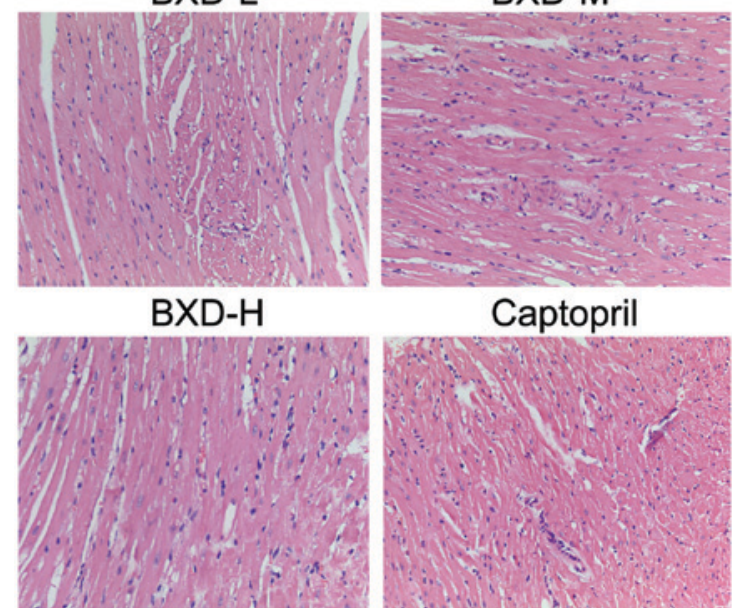

Captopril

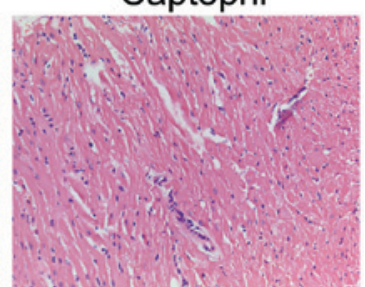

Figure 2. Effect of BXD on myocytes. Representative of left ventricular sections of hearts from different experimental groups, stained with H\&E (magnification, x200). DCM, dilated cardiomyopathy; BXD, baoxin decoction; $\mathrm{H} \& \mathrm{E}$, hematoxylin and eosin.

\section{Results}

BXD improves cardiac function in rats with DCM. In order to determine whether BXD improved cardiac function, we performed echocardiography 4 weeks post-treatment. Echocardiographic parameters of the respective groups of rats are shown in Table I. It shows a representative echocardiography view of hearts from each group after treatment. As shown in Table I, echocardiography analyses after treatment indicated that compared to those in the $\mathrm{CON}$ group, the rats in the DCM, BXD and captopril groups exhibited significant left ventricular dilation and systolic dysfunction. The LVEDD and LVESD were significantly increased while the LVEF and LVFS were significantly decreased $(\mathrm{P}<0.01$, Fig. 1). The LVESD, LVEDD, LVEF and LVFS in the BXD and 


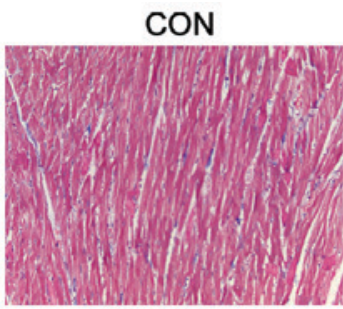

BXD-M

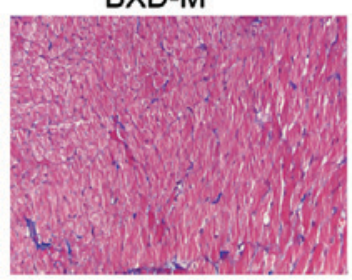

DCM

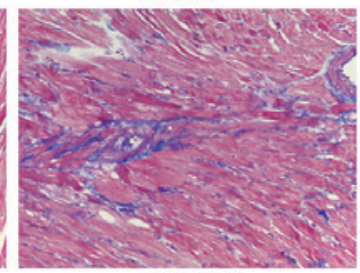

BXD-H

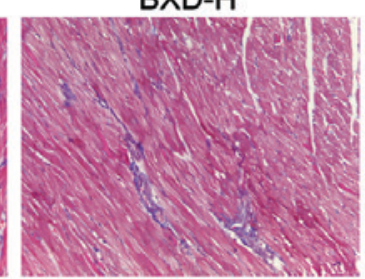

BXD-L

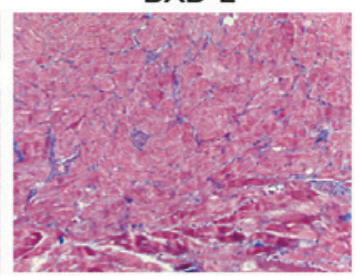

Captopril

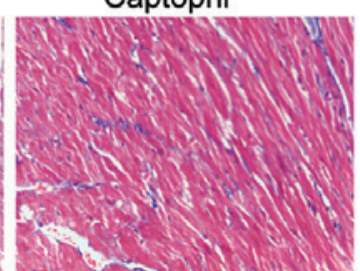

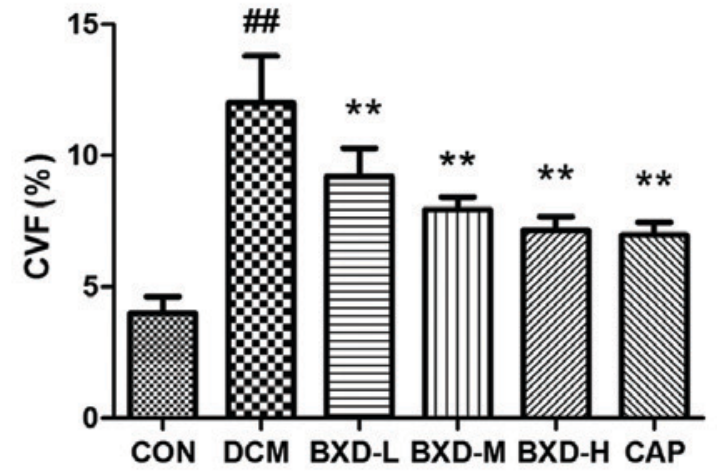

Figure 3. Effect of BXD on myocardial fibrosis. Representative of left ventricular sections of hearts from the different experimental groups, stained with Masson's trichrome staining (magnification, $\mathrm{x} 200$ ). BXD, baoxin decoction; DCM, dilated cardiomyopathy; ${ }^{\# \#} \mathrm{P}<0.01 \mathrm{vs}$. control group; ${ }^{* *} \mathrm{P}<0.01 \mathrm{vs}$. DCM group.
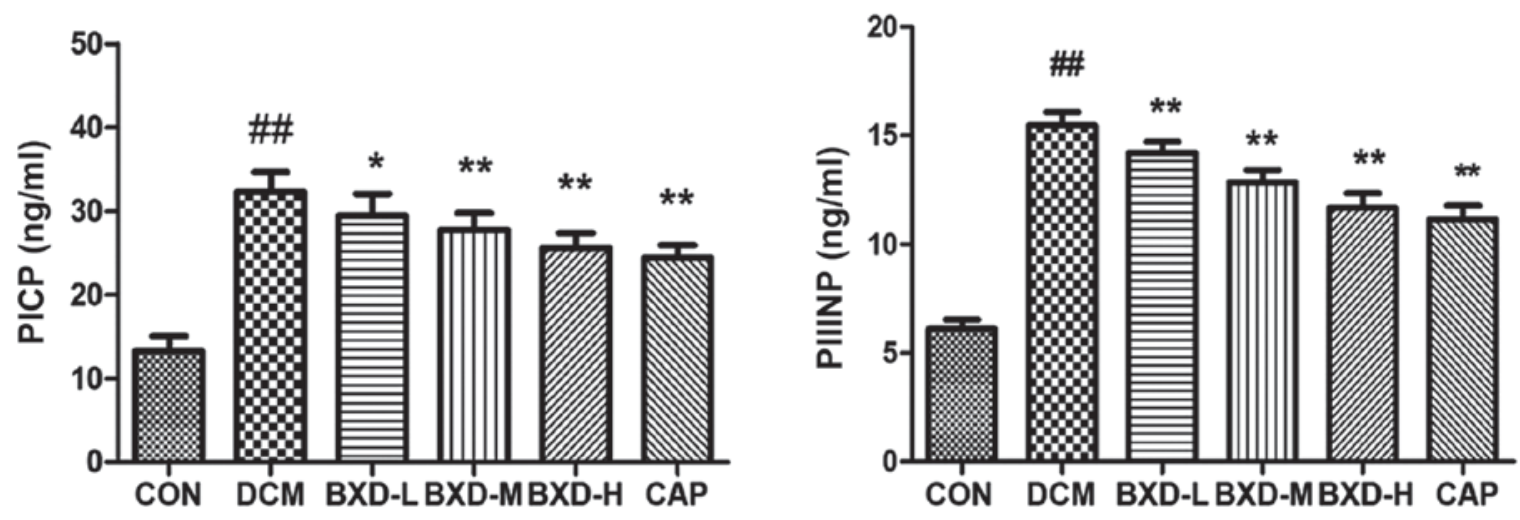

Figure 4. Effect of BXD on serum biochemical markers of myocardial fibrosis. The quantitative data of carboxy-terminal propeptides of collagens I (PICP) and amino-terminal propeptides of collagens III (PIIINP). Results are expressed as mean \pm SD. ${ }^{\#} \mathrm{P}<0.01$ vs. control group; ${ }^{*} \mathrm{P}<0.05$, ${ }^{* *} \mathrm{P}<0.01 \mathrm{vs}$. DCM group; BXD, baoxin decoction; PICP, propeptides of collagens I; PIIINP, propeptides of collagens III; DCM, dilated cardiomyopathy.

captopril-treated group were significantly improved after treatment when compared to the DCM group ( $\mathrm{P}<0.01$, Fig. 1), demonstrating that BXD treatment markedly ameliorated cardiac dysfunction.

BXD attenuates DCM rat myocardial pathological change. Fig. 2 shows the H\&E-stained sections of left ventricular myocardial tissue with the control group showing normal histomorphology. Tissues from the DCM group showed enhancement and loose arrangement of the myocardial fibers, hypertrophy, myocardial necrosis, loss of myocytes and vacuolar degeneration. Administration of BXD and captopril improved cardiac hypertrophy and alleviated areas of necrosis. As Fig. 3 shows, Masson's trichrome staining for interstitial fibrosis on the left ventricular myocardial tissue. The areas of fibrosis (blue) show CVF. CVF of the DCM group were increased $(\mathrm{P}<0.001)$ when compared to the control group. The treatment with either BXD or captopril was associated with a reduction in CVF (BXD and captopril, $\mathrm{P}<0.01$ ).

BXD inhibits myocardial collagen. The carboxy-terminal propeptides of collagen I (PICP) and amino-terminal propeptides of collagen III (PIIINP) are serum biochemical markers of cardiac ECM. As Fig. 4 shows, when compared to the control group, the levels of serum PICP and PIIINP were significantly increased in the DCM group $(\mathrm{P}<0.01)$. BXD and captopriltreated group decreased the concentration of serum PICP and PIIIN ( $\mathrm{P}<0.05-0.01$ vs. the DCM group). These data indicated that BXD played a protective role in DCM by decreasing the synthesis and degradation of myocardial collagen.

Effects of BXD on heart tissue Gal-3, Col I and Col III mRNA expression. Gal-3, Col I and Col III mRNA levels in the left ventricles myocardial were significantly increased in the DCM group compared to the control group $(\mathrm{P}<0.01$, Fig. 5). As expected, treatment with either BXD or captopril was associated with a significantly lower expression of Gal-3, Col I and Col III $(\mathrm{P}<0.01)$.

Effects of BXD on heart tissue Gal-3, TGF- $\beta 1$ and Smad3 protein expression. Gal-3 protein expression levels and molecules involved in the Gal-3 signaling pathway (TGF- $\beta$ and Smad3) were analyzed by western blot analysis for each experimental group. It is well known that TGF- $\beta 1 / \mathrm{Smad} 3$ signaling is a key pathway in the myocardial fibrosis process. Results indicated that BXD significantly reduced Gal-3, TGF- $\beta 1$ and 
A

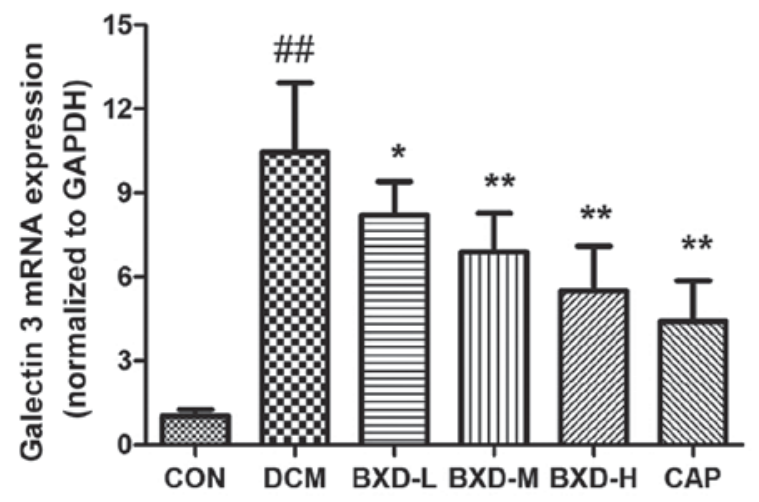

B

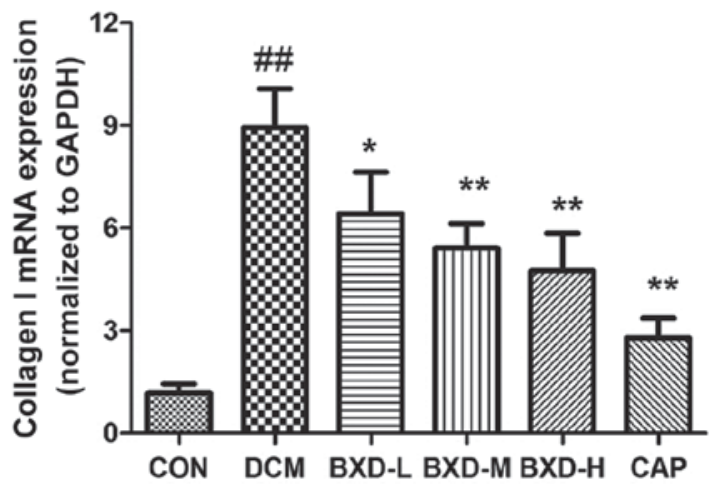

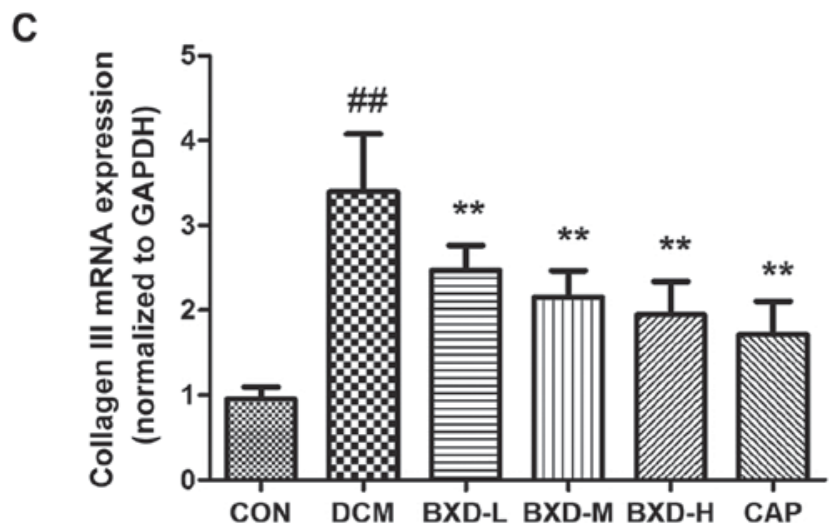

Figure 5. Expression of Gal-3, Col I and Col III mRNA in the ventricle of DCM rats and the effect of BXD. RT-qPCR was performed. (A) Gal-3. (B) Col I. (C) Col III. Results are expressed as mean \pm SD. ${ }^{\# \#} \mathrm{P}<0.01$ vs. control group. ${ }^{*} \mathrm{P}<0.05,{ }^{* *} \mathrm{P}<0.01$ vs. DCM group; DCM, dilated cardiomyopathy; Gal-3, galectin-3; BXD, baoxin decoction; Col I, collagen I; Col III, collagen III.

A
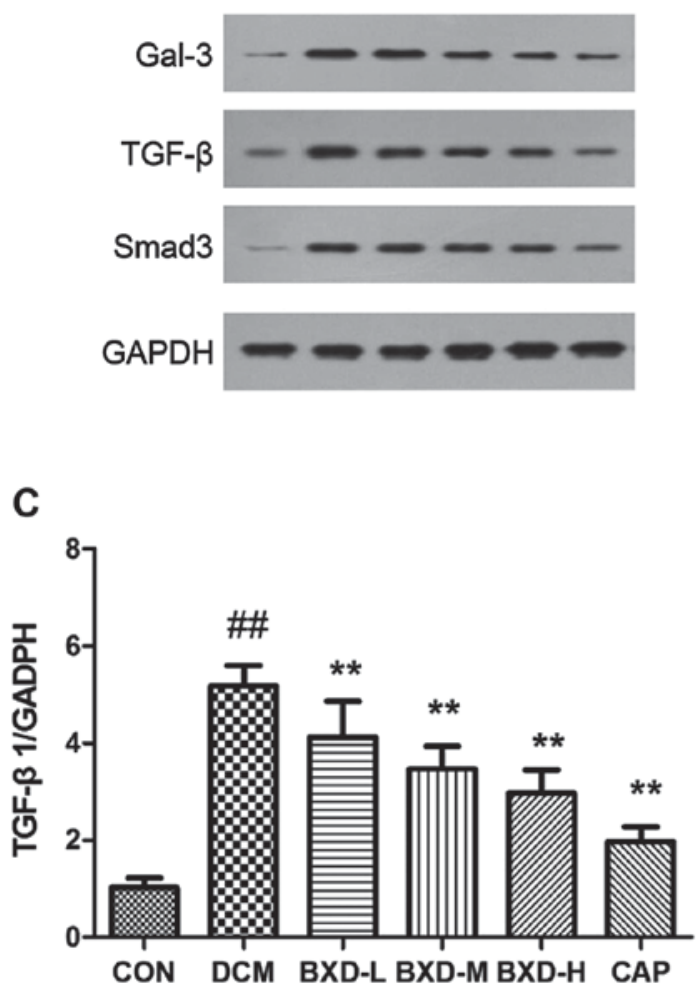

B

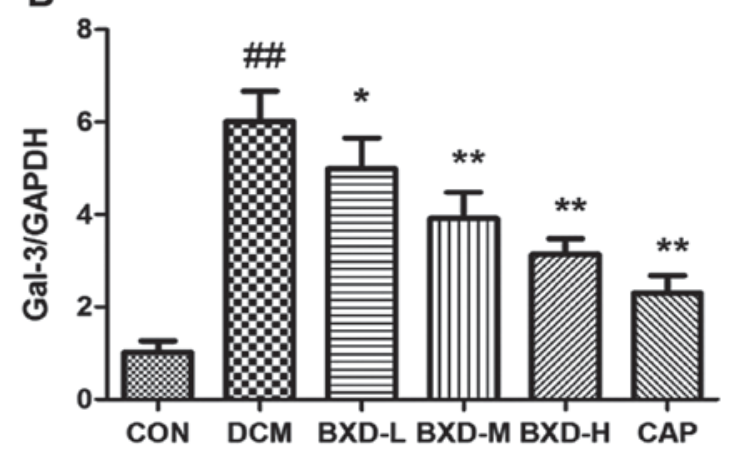

D

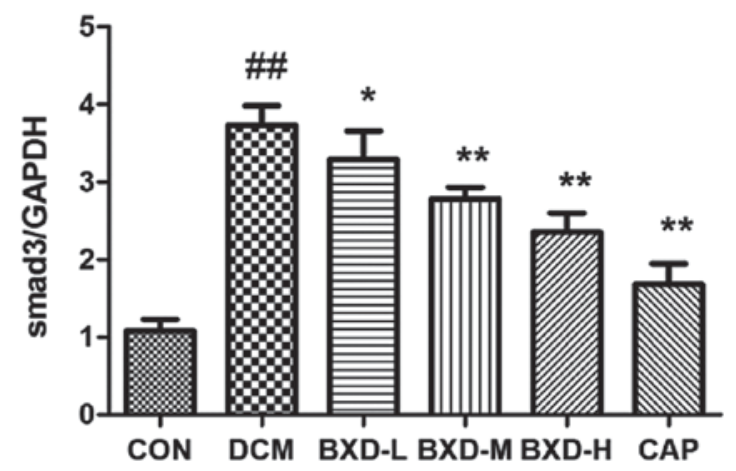

Figure 6. Effect of BXD on TGF- $\beta 1 /$ Smad3 signaling expression. (A) Representative western blot analysis of myocardial fibrosis tissue. (B-D) The ratios of various proteins to GAPDH. Protein levels were calculated by densitometry. Results are expressed as mean $\pm \mathrm{SD}$. ${ }^{\# \#} \mathrm{P}<0.01$ vs. control group, ${ }^{*} \mathrm{P}<0.05$, ${ }^{* *} \mathrm{P}<0.01$ vs. DCM group; BXD, baoxin decoction; DCM, dilated cardiomyopathy; Gal-3, galectin-3. 
Smad-3 protein expression $(\mathrm{P}<0.05, \mathrm{P}<0.01$, Fig. 6). Notably, the inhibitory effect of BXD on myocardial fibrosis signaling was associated with the downregulation of TGF- $\beta 1 / \mathrm{Smad} 3$ signaling.

\section{Discussion}

In this study, we investigated the effects of BXD on myocardial dysfunction, fibrosis and interrelated signaling pathways using a rat model of doxorubicin-induced DCM. The main findings of this study include that BXD reduced left ventricular dilation and improved left ventricular systolic function in rats of doxorubicin-induced DCM, BXD effectively attenuated interstitial fibrosis by inhibiting collagen production and the underlying mechanism may be associated with the suppression of Gal-3 and TGF- $\beta 1 /$ Smad3 signaling.

Doxorubicin is used as a common chemotherapeutic agent, which can also lead to cardiotoxicity. Many experimental animal models of Dox-induced cardiomyopathy have been used to investigate DCM (13). In this study, we followed the animal model of DCM. Echocardiography showed significant dilation of the left ventricle and a significant reduction of cardiac function. Pathological examination showed a large accumulation of collagen in the myocardial interstitial tissues. Our results demonstrated that this model is successful, which is in agreement with previous findings (14).

A number of previous reports have described herbal medicines as being effective in preventing or decreasing myocardial fibrosis in cardiovascular disease. Evidence gathered from a systematic review shows that herbal medicine, which seems to be relatively safe and convenient, may offer a much needed alternative and merit further attention (15). BXD is a specific TCM that has been developed based on the meridian theory. Therefore, we evaluated the effects of BXD in an animal experimental model on myocardial dysfunction, fibrosis and interrelated signaling pathways.

Gal-3 is a member of the galectin family, and consists of animal lectins that bind $\beta$-galactosides (16). In recent years, Gal-3 has emerged as a link to the pathophysiology of adverse myocardial remodeling (17). This has been associated with activation of fibroblasts and macrophages, which leads to the development of interstitial and perivascular fibrosis and left ventricular dysfunction (18). Some studies have indicated that inhibition of Gal-3 was associated with a downregulation in collagen production. Furthermore, the inhibition of Gal-3 has also been shown to attenuate the progression of cardiac remodeling in a long-term transverse aortic constriction mouse model (19). Ac-SDKP has been known to prevent interstitial and perivascular fibrosis and LV dysfunction was caused by Gal-3. These changes were shown to be mediated by a transforming growth factor TGF- $\beta /$ Smad3 pathway (20). The mineralocorticoid receptor antagonists eplerenone and spironolactone, modulated Gal- 3 and TGF- $\beta /$ Smad 3 signaling in an experimental model of left ventricular systolic dysfunction (21). These findings suggest that therapeutic agents targeting Gal-3 might result in innovative new therapies. In our study, BXD treatment was associated with an inhibition of the observed upregulation of Gal-3.

Myocardial fibrosis is one of the main pathological changes in DCM. The TGF- $\beta$-Smad3 pathway contributes to the long progress of myocardial fibrosis. TGF- $\beta 1$ plays a crucial role in cardiac fibrosis by activating fibroblasts and producing collagen (22). TGF- $\beta 1$ inhibition of cardiac fibroblast proliferation requires Smad3 (23). Therefore, inhibiting Smad3 transduction may prevent cardiac fibrosis. As shown in some studies, inhibiting the TGF- $\beta 1$-Smad3 signaling pathway or modulating the gene expression of Smad 3 could effectively interfere with myocardial fibrosis (24-26). Many Chinese herbal medicines can inhibit cardiac fibrosis, such as Gualou Xiebai decoction, by blocking TGF- $\beta 1 /$ Smad3 signaling (27). Shensong Yangxin capsule prevents diabetic myocardial fibrosis by inhibiting TGF- $\beta 1 /$ Smad 3 signaling (28). Captopril, an ACE inhibitor, prevents the development and progression of subsequent fibrosis related to the reduction of TGF- $\beta 1$ levels. The findings of our study show that BXD may possibly be able to bring about similar effects in myocardial fibrosis associated with Gal-3 and TGF- $\beta$-Smad 3 pathway.

However, the present findings of the study are limited by the experiment. The relationship between cardiac fibrosis and Gal-3 and TGF- $\beta / \operatorname{smad} 3$ has not demonstrated causality. In addition, the mechanism of BXD needs to be further explored.

\section{References}

1. Gopal DM and Sam F: New and emerging biomarkers in left ventricular systolic dysfunction - Insight into dilated cardiomyopathy. J Cardiovasc Transl Res 6: 516-527, 2013.

2. Towbin JA, Lowe AM, Colan SD, Sleeper LA, Orav EJ, Clunie S, Messere J, Cox GF, Lurie PR, Hsu D, et al: Incidence, causes, and outcomes of dilated cardiomyopathy in children. JAMA 296: 1867-1876, 2006.

3. Luk A, Ahn E, Soor GS and Butany J: Dilated cardiomyopathy: A review. J Clin Pathol 62: 219-225, 2009.

4. Carasso S and Amir O: Reverse remodeling in dilated cardiomyopathy: Dream come true? Isr Med Assoc J 16: 444-445, 2014.

5. Nicolini G, Pitto L, Kusmic C, Balzan S, Sabatino L, Iervasi G and Forini F: New insights into mechanisms of cardioprotection mediated by thyroid hormones. J Thyroid Res 2013: 264387, 2013.

6. Jalil JE, Doering CW, Janicki JS, Pick R, Shroff SG and Weber KT: Fibrillar collagen and myocardial stiffness in the intact hypertrophied rat left ventricle. Circ Res 64: 1041-1050, 1989.

7. Kong P, Christia P and Frangogiannis NG: The pathogenesis of cardiac fibrosis. Cell Mol Life Sci 71: 549-574, 2014.

8. Graf K and Schaefer-Graf UM: Is Smad3 the key to inflammation and fibrosis in hypertensive heart disease? Hypertension 55: 1088-1089, 2010.

9. Ferreira AS and Lopes AJ: Chinese medicine pattern differentiation and its implications for clinical practice. Chin J Integr Med 17: 818-823, 2011.

10. Xiang P, Deng HY, Li K, Huang GY, Chen Y, Tu L, Ng PC, Pong $\mathrm{NH}$, Zhao $\mathrm{H}$, Zhang L, et al: Dexrazoxane protects against doxorubicin-induced cardiomyopathy: Upregulation of Akt and Erk phosphorylation in a rat model. Cancer Chemother Pharmacol 63: 343-349, 2009.

11. Shen FF, Jiang TH, Jiang JQ, Lou Y and Hou XM: Traditional chinese medicine tongxinluo improves cardiac function of rats with dilated cardiomyopathy. Evid Based Complement Alternat Med 2014: 323870, 2014.

12. Qin YW, Ye P, He JQ, Sheng L, Wang LY and Du J: Simvastatin inhibited cardiac hypertrophy and fibrosis in apolipoprotein E-deficient mice fed a 'Western-style diet' by increasing PPAR $\alpha$ and $\gamma$ expression and reducing TC, MMP-9, and Cat S levels. Acta Pharmacol Sin 31: 1350-1358, 2010.

13. Feng Y, Xu H and Chen K: Natural Polypill Xuezhikang: Its clinical benefit and potential multicomponent synergistic mechanisms of action in cardiovascular disease and other chronic conditions. J Altern Complement Med 18: 318-328, 2012.

14. Hayward R and Hydock DS: Doxorubicin cardiotoxicity in the rat: an in vivo characterization. J Am Assoc Lab Anim Sci 46: 20-32, 2007. 
15. Schwarz ER, Pollick C, Dow J, Patterson M, Birnbaum Y and Kloner RA: A small animal model of non-ischemic cardiomyopathy and its evaluation by transthoracic echocardiography. Cardiovasc Res 39: 216-223, 1998

16. Barondes SH, Cooper DN, Gitt MA and Leffler H: Galectins. Structure and function of a large family of animal lectins. J Biol Chem 269: 20807-20810, 1994.

17. Shah RV and Januzzi JL Jr: Soluble ST2 and galectin-3 in heart failure. Clin Lab Med 34: 87-97, 2014

18. Wu AH, Wians F and Jaffe A: Biological variation of galectin-3 and soluble ST2 for chronic heart failure: Implication on interpretation of test results. Am Heart J 165: 995-999, 2013.

19. Yu L, Ruifrok WP, Meissner M, Bos EM, van Goor H, Sanjabi B, van der Harst P, Pitt B, Goldstein IJ, Koerts JA, et al: Genetic and pharmacological inhibition of galectin-3 prevents cardiac remodeling by interfering with myocardial fibrogenesis. Circ Heart Fail 6: 107-117, 2013.

20. Sharma UC, Pokharel S, van Brakel TJ, van Berlo JH, Cleutjens JP, Schroen B, André S, Crijns HJ, Gabius HJ, Maessen J, et al: Galectin-3 marks activated macrophages in failure-prone hypertrophied hearts and contributes to cardiac dysfunction. Circulation 110: 3121-3128, 2004.

21. Lax A, Sanchez-Mas J, Asensio-Lopez MC, FernandezDel Palacio MJ, Caballero L, Garrido IP, Pastor-Perez FJ, Januzzi JL and Pascual-Figal DA: Mineralocorticoid receptor antagonists modulate galectin-3 and interleukin-33/ST2 signaling in left ventricular systolic dysfunction after acute myocardial infarction. JACC Heart Fail 3: 50-58, 2015.
22. Du J, Xie J, Zhang Z, Tsujikawa H, Fusco D, Silverman D, Liang B and Yue L: TRPM7-mediated $\mathrm{Ca}^{2+}$ signals confer fibrogenesis in human atrial fibrillation. Circ Res 106: 992-1003, 2010.

23. Dobaczewski M, Bujak M, Li N, Gonzalez-Quesada C, Mendoza LH, Wang XF and Frangogiannis NG: Smad3 signaling critically regulates fibroblast phenotype and function in healing myocardial infarction. Circ Res 107: 418-428, 2010.

24. Jia N, Dong P, Ye Y, Qian C and Dai Q: Allopurinol attenuates oxidative stress and cardiac fibrosis in angiotensin II-induced cardiac diastolic dysfunction. Cardiovasc Ther 30: 117-123, 2012.

25. Xiao H, Ma X, Feng W, Fu Y, Lu Z, Xu M, Shen Q, Zhu Y and Zhang Y: Metformin attenuates cardiac fibrosis by inhibiting the TGF $31-$ Smad3 signalling pathway. Cardiovasc Res 87: 504-513, 2010

26. Zhao M, Zheng S, Yang J, Wu Y, Ren Y, Kong X, Li W and Xuan J: Suppression of TGF- $\beta 1 /$ Smad signaling pathway by sesamin contributes to the attenuation of myocardial fibrosis in spontaneously hypertensive rats. PLoS One 10: e0121312, 2015.

27. Ding YF, Peng YR, Li J, Shen H, Shen MQ and Fang TH: Gualou Xiebai Decoction prevents myocardial fibrosis by blocking TGF- $3 /$ Smad signalling. J Pharm Pharmacol 65: 1373-1381, 2013.

28. Shen N, Li X, Zhou T, Bilal MU, Du N, Hu Y, Qin W, Xie Y, Wang H, Wu J, et al: Shensong Yangxin Capsule prevents diabetic myocardial fibrosis by inhibiting TGF- $\beta 1 /$ Smad signaling. J Ethnopharmacol 157: 161-170, 2014. 